\title{
Screening in Anyon Gas
}

\author{
Subir Ghosh \\ Physics Department Dinabandhu Andrews College, Calcutta 700084, India
}

\begin{abstract}
Anyon gas with interparticle (retarded) Coulomb interaction has been studied. The resulting system is shown to be a collection of dressed anyons, with a screening factor introduced in their spin. Close structural similarity with the Chern-Simons construction of anyons has helped considerably in computing the screening effect. Finally the present model is compared with the conventional Chern-Simons construction.
\end{abstract}


The possible existence of particles having arbitrary spin and statistics has been proved quite some time back [1, 2]. However, dynamical model building, on the other hand, has proved to be rather controversial, with the dispute still continuing. Basically there are two broad lines along which the models are conceived: (i) The Chern-Simons (CS) construction [3], where a point charge is coupled to CS eletrodynamics. Removal of the auxiliary (or statistical) CS gauge field renders the particle anyonic. (ii) The construction of minimal anyon field equations, where one starts from very general physical postulates, such as the mass shell and Pauli-Lubanski condition for the particle [4]. A variant of the latter scheme is the spinning particle model [5, 6, 7], with which we are concerned in the present Letter. The connection between the latter two is elaborated in [ $[$ ]

It is important to point out that individually both models represent anyons. The controversy arises as regards the nature of the CS gauge field in (i). The contention of [3], that the only effect of the CS gauge field is to influence the particle statistics and nothing else, has been debated strongly in [9]. Also the CS scheme fails in the relativistic theory, relevant for cosmic string problems.

Our result in this Letter show conclusively that anyons in the presence of genuine interparticle Coulomb interaction, are dressed as far as their fractional spin (and hence the statistics parameter) is concerned. The screened spin $S=\alpha j$ is $\alpha=-\frac{Q^{2}}{16 \pi^{2} \epsilon_{0} m c^{2}}$, whereas in the spinning partcle models [5], [6], the spin is $S=j$, where $j$ is the Lagrangian spin parameter in (11). Here $Q$ and $m$ are the charge and mass of the anyon, $c$ the velocity of light and $\epsilon_{0}$ a characteristic property of the vacuum, (to be elaborated later). The dimensionless quantity $\alpha$ is the screening factor. We have considered a two particle system but generalization to a many particle system is straightforward.

Also the other interesting feature of the model is its structural similarity with CS construction [2]. We show that in the slow moving and large mass particle limit, the Coulomb field is structurally identical to the CS gauge field solution in [2], with the identification of the CS $\theta$ parameter, $\theta=-\frac{4 \pi \epsilon_{0} m c^{2}}{j}$. The crucial difference lies in the qualitative nature of the CS gauge field and the Coulomb field considered here. The former is sort of a fictitous gauge field [10], that couples to the fictitous charge of the particle, whereas the latter is the real Coulomb field, responsible for the Lorentz force between particles. Apart from this, there is the usual logarithmic Coulomb potential. Note that in conventional CS scheme, the particles are endowed with the fractional spin $S=\frac{Q^{2}}{4 \pi \theta_{C S}}$ where $\theta_{C S}$ is the arbitrary CS parameter and $Q$ is the fictional charge that couples to 
the CS statistical gauge field. One recovers the logarithmic Coulomb interaction as well [10]. The above mentioned identification helps us to make use of the CS results directly to compute explicitly the screening factor $\alpha$. The connection between conventional CS scheme and our model will be elaborated at the end. Although, some of the major results of this paper have appeared in [11], the implications and consequences, discussed in the conclusion, were not emphasized before.

The spinning particle Lagrangian proposed by us in [6] is, $(c=1)$,

$$
L=\sqrt{m^{2} u^{2}+\frac{1}{2} j^{2} \sigma^{2}+m j \epsilon^{\mu \nu \lambda} u_{\mu} \sigma_{\nu \lambda}},
$$

where the velocity and canonical momenta are defined as

$$
\begin{gathered}
u^{\mu}=\frac{d r^{\mu}}{d \tau} ; \quad \sigma^{\mu \nu}=\Lambda_{\lambda}{ }^{\mu} \dot{\Lambda}^{\lambda \nu}, \\
P^{\mu}=-\frac{\partial L}{\partial u_{\mu}} ; \quad S^{\mu \nu}=-\frac{\partial L}{\partial \sigma_{\mu \nu}} .
\end{gathered}
$$

$\left(r^{\mu}, \Lambda^{\mu, \nu}\right)$ is a Poincare group element, as well dynamical variables with the property, $\Lambda \Lambda^{T}=\Lambda^{T} \Lambda=g$, where $g$ is the Minkowski metric $g^{00}=-g^{11}=-g^{22}=1$.

The action in (1), $\int L d \tau$ is invariant under reparametrizations of the arbitrary parameter $\tau \rightarrow \tau^{\prime}=f(\tau)$. The details of the constraint analysis can be found in [6]. We will use the relevant Dirac Brackets (DB) as and when necessary. Let us briefly demonstrate the appearence of the arbitrary phase. The set of Second Class Constraints (SCC) and First Class Constraints (FCC) are

$$
\begin{gathered}
S^{\mu \nu} P_{\nu} \approx 0 ; \quad \Lambda^{0 \mu}-\frac{P^{\mu}}{m} \approx 0, \\
P^{2}-m^{2} \approx 0 ; \quad \epsilon^{\mu \nu \lambda} S_{\mu \nu} P_{\lambda}-m j \approx 0 .
\end{gathered}
$$

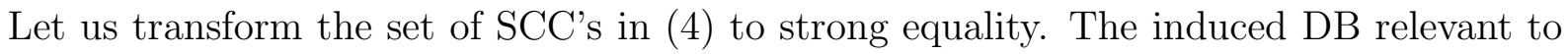
us,

$$
\begin{gathered}
\left\{\Lambda^{\mu \nu}, S^{12}\right\}=\left(\Lambda^{\mu 1} g^{\nu 2}-\Lambda^{\mu 2} g^{\nu 1}\right) \\
+\frac{1}{m^{2}}\left(P^{\nu} P^{1} \Lambda^{\mu 2}-P^{\nu} P^{2} \Lambda^{\mu 1}-P_{\rho} \Lambda^{\mu \rho} P^{1} g^{\nu 2}+P_{\rho} \Lambda^{\mu \rho} P^{2} g^{\nu 1}\right),
\end{gathered}
$$

in the particle rest frame, $P^{i}=0, P^{0}=m$, reduces to

$$
\begin{aligned}
& \left\{\Lambda^{11}, S^{12}\right\}=\Lambda^{12} ; \quad\left\{\Lambda^{22}, S^{12}\right\}=-\Lambda^{21} \\
& \left\{\Lambda^{12}, S^{12}\right\}=-\Lambda^{11} ; \quad\left\{\Lambda^{21}, S^{12}\right\}=\Lambda^{22}
\end{aligned}
$$


Using the rest frame $\Lambda^{\prime}$ s, ie. $\Lambda^{01}=\Lambda^{02}=0, \quad \Lambda^{00}=1$ we get the relations,

$$
\begin{array}{cc}
\Lambda^{10}=\Lambda^{20}=0 ; & \left(\Lambda^{12}\right)^{2}+\left(\Lambda^{11}\right)^{2}=\left(\Lambda^{21}\right)^{2}+\left(\Lambda^{22}\right)^{2}=1 \\
& \Lambda^{11} \Lambda^{21}+\Lambda^{12} \Lambda^{22}=0
\end{array}
$$

Hence in the reduced phase space we can parametrize the remaining independent variables by,

$$
\Lambda^{12}=\cos \phi ; \quad \Lambda^{11}=\sin \phi ; \quad S^{12}=\frac{\partial}{\partial \phi}
$$

where $S^{12}$ is the Pauli-Lubanski scalar in the rest frame,

$$
\left.\frac{\epsilon^{\mu \nu \lambda} S_{\mu \nu} P_{\lambda}}{m}\right|_{\text {rest frame }}=\frac{S_{12} P_{0}}{m}=S_{12} .
$$

Also from counting the number of independent degrees of freedom in phase space we see that out of three each (independent) $S^{\mu \nu}$ and $\Lambda^{\mu \nu}$ variables one each of $S$ and $\Lambda$ remain, since out of the set of six SCC's in (乐) only two from each set (totalling four) are independent. So far the FCC's have remained intact. This is exactly the parametrization employed by Plyushchay in [5]. This $\Lambda$ variable, (or equivalently $\phi$ ), gives rise to the arbitrary phase. This is consistent with the fact [12] that specifically in 2+1-dimensions, the number of (phase space) degrees of freedom for a particle with fixed mass and spin is the same as that of a massive spinless particle. Here the remaining degrees of freedom, ie. $\phi$ and $S^{12}$, can be removed by choosing a gauge for the Pauli-Lubanski FCC. However, the effect of the spin variables present in (11) manifest itself in the non-trivial DB's, which gives rise to the spin contribution in the total angular momentum.

Note an interesting departure in the constraint structure from the parent $3+1$-dimensional model [13], where the spin (FC) constraint appeared as a combination of the SCC's $S^{\mu \nu} P_{\nu} \equiv 0$, due to some non-trivial algebraic identities. The latter are absent in $2+1$ dimensions, as a result of which the spin constraint comes here independently, ie. it is not obtainable from the other FCC and SCC's, in (4) and (5).

Let us now move to the main body of our work. From now on we will use rationalized MKS units and keep $c$ and $\hbar$ as they come. This will be useful for the comparison of the final results and churning out numerical estimates. We use the planar Coulomb law as

$$
\mathbf{F}_{\text {Coul }}=\frac{Q_{1} Q_{2}}{2 \pi \epsilon_{0} r} \mathbf{n}
$$

where $\mathbf{r}=r \mathbf{n}$ is the separation between the charges $Q_{1}$ and $Q_{2}$, and $\epsilon_{0}$ is the "permittivity" of the vacuum. $\mathbf{F}_{\text {Coul }}$ denotes the force between the particles. This Coulomb law is 
compatible with the Gauss law in a plane, $\nabla . \mathbf{E}=\frac{\rho}{\epsilon_{0}}$, where $\mathbf{E}$ and $\rho$ are the electric field and charge density respectively. We introduce $\mu_{0}$ and $\epsilon_{0}$ as the "permeability" and "permittivity" of the vacuum, to keep the relations same as their 3+1-dimensional counterpart. We only use the relation $\epsilon_{0} \mu_{0}=\frac{1}{c^{2}}$. Denoting by $[O]=$ dimension of $O$, we note that

$$
\left[\epsilon_{0}\right]=\frac{C^{2}}{M(L / T)^{2}} ; \quad[\phi]=\frac{M(L / T)^{2}}{C} ; \quad\left[A_{i}\right]=\frac{M(L / T)}{C} .
$$

Here $M, L, T, C$ are mass, length, time and Coulomb. $\phi$ and $A_{i}$ are the scalar and vector potentials. We have the standard relations,

$$
\mathbf{E}=-\nabla \phi-\dot{\mathbf{A}}, \mathbf{B}=\nabla X \mathbf{A}
$$

Here $\mathbf{B}$ is the magnetic field.

We briefly show the construction of the relativistic Darwin Lagrangian for a system of two interacting point charges in a plane. As the results have appeared in [11], we simply incorporate $c, \epsilon_{0}$ and $\mu_{0}$ in their respective places. The retarded logarithmic potentials are,

$$
\begin{aligned}
& \phi=\frac{1}{2 \pi \epsilon_{0}} \int d^{2} r \rho\left(\mathbf{r}, t-\frac{r}{c}\right) \ln \frac{r}{r_{0}}, \\
& \mathbf{A}=\frac{\mu_{0}}{2 \pi} \int d^{2} r \rho\left(\mathbf{r}, t-\frac{r}{c}\right) \mathbf{v} \ln \frac{r}{r_{0}} .
\end{aligned}
$$

$r_{0}$ denotes some length scale where the potential due to a point charge vanishes. Expanding in terms of $v$-the particle velocity and keeping terms upto $O\left(\frac{v^{2}}{c^{2}}\right)$, with the charge density $\rho=Q \delta\left(\mathbf{r}-\mathbf{r}_{\text {particle }}\right)$, we get

$$
\begin{gathered}
\phi=\frac{Q}{2 \pi \epsilon_{0}}\left[\ln \frac{r}{r_{0}}-\frac{1}{c}\left(r \ln \frac{r}{r_{0}} j+\frac{1}{2 c^{2}}\left(r^{2} \ln \frac{r}{r_{0}}\right)\right],\right. \\
\mathbf{A}=\frac{\mu_{0}}{2 \pi} Q \mathbf{v} \ln \frac{r}{r_{0}} .
\end{gathered}
$$

Performing a gauge transformation,

$$
\phi \rightarrow \phi^{\prime}=\phi-\frac{\partial f}{\partial t} ; \quad \mathbf{A} \rightarrow \mathbf{A}^{\prime}=\mathbf{A}+\nabla f
$$

such that,

$$
\begin{gathered}
\frac{\partial f}{\partial t}=\frac{Q}{2 \pi \epsilon_{0}}\left[-\frac{1}{c}\left(r \ln \frac{r}{r_{0}} \dot{)}+\frac{1}{2 c^{2}}\left(r^{2} \ln \frac{r}{r_{0}}\right)\right]\right. \\
\nabla f=\frac{Q}{2 \pi \epsilon_{0}}\left(-\frac{\mathbf{n}}{c} \ln \frac{r}{r_{0}}-\frac{\mathbf{n}}{c}\right)+\frac{Q}{4 \pi \epsilon_{0} c^{2}}\left(2 \mathbf{n} r \ln \frac{r}{r_{0}}+\mathbf{r}\right)
\end{gathered}
$$


the retarded potential $\phi$ is reduced to the standard Coulomb form,

$$
\begin{gathered}
\phi^{\prime}=\frac{Q}{2 \pi \epsilon_{0}} \ln \frac{r}{r_{0}} \\
\mathbf{A}^{\prime}=-\frac{Q}{2 \pi \epsilon_{0}}\left[\frac{\mathbf{n}}{c^{2}}\left(\mathbf{n} \cdot \mathbf{v}+c\left(1+\ln \frac{r}{r_{0}}\right)\right)+\frac{\mathbf{v}}{c^{2}}\right] .
\end{gathered}
$$

The interaction is simply of the minimal current-gauge field form $J_{\mu} A^{\prime \mu}$, where $J_{0}=\rho=$ $Q \delta\left(\mathbf{r}-\mathbf{r}_{p}\right), \mathbf{J}=Q \mathbf{v} \delta\left(\mathbf{r}-\mathbf{r}_{p}\right)$ and $A^{\prime \mu}$ is the above set, (13) and (14). Thus, to $O\left(\frac{v^{2}}{c^{2}}\right)$, the Lagrangian, or the Hamiltonian obtained just below, incorporate the effect of Coulomb interaction between two charges, taking into account the relativistic corrections via the retarded time.

The two-particle Darwin Hamiltonian is,

$$
H=\frac{p^{2}}{m}-\frac{p^{4}}{4 m^{3} c^{2}}+\frac{Q^{2}}{2 \pi \epsilon_{0}} \ln \frac{r}{r_{0}}+\frac{Q^{2}}{2 \pi \epsilon_{0} c^{2}}\left[\frac{\mathbf{r} \cdot \mathbf{p}}{m r}\left(\frac{\mathbf{r} \cdot \mathbf{p}}{m r}+c\left(1+\ln \frac{r}{r_{0}}\right)\right)-\frac{p^{2}}{2 m^{2}}\right] .
$$

Note that the correction terms in $\mathbf{A}^{\prime}$ are qualitatively different from their 3+1-dimensional counterpart [14]. This has induced difference in the Hamiltonian correction terms as well.

So far the effect of the particle spin has not been taken into account. Now we do this via a non-canonical transformation. We rewrite (11),

$$
L=c^{2} \sqrt{\left(\frac{m^{2} u^{2}}{c^{2}}+\frac{j^{2} \sigma^{2}}{2 c^{4}}+\frac{m j}{c^{3}} \epsilon^{\mu \nu \lambda} u_{\mu} \sigma_{\nu \lambda}\right)},
$$

with the dimensions of the phase space variables being,

$$
[u]=\frac{L}{T} ; \quad[\sigma]=[\Lambda \dot{\Lambda}]=T^{-1} ; \quad\left[S_{\mu \nu}\right]=[j]=\frac{M L^{2}}{T} ; \quad\left[P_{\mu}\right]=\frac{M L}{T} .
$$

With $P^{2}=m^{2} c^{2}$ and $S^{2}=2 j^{2}$, the DB's relevant to us are [6],

$$
\left\{r^{\mu}, r^{\nu}\right\}=-\frac{S^{\mu \nu}}{m^{2} c^{2}}=-\frac{j}{m^{3} c^{3}} \epsilon^{\mu \nu \lambda} P_{\lambda} ; \quad\left\{r^{\mu}, P^{\nu}\right\}=g^{\mu \nu} ; \quad\left\{P^{\mu}, P^{\nu}\right\}=0 .
$$

Invoking the quantization prescription that $\frac{i}{\hbar}\{D B\} \rightarrow$ [commutator], we arrive at the following commutators,

$$
\left[r^{\mu}, r^{\mu}\right]=\frac{i \hbar}{m^{2} c^{2}} S^{\mu \nu}=\frac{i \hbar j}{m^{3} c^{3}} \epsilon^{\mu \nu \lambda} P_{\lambda} ; \quad\left[r^{\mu}, P^{\nu}\right]=-i \hbar g^{\mu \nu} ; \quad\left[P^{\mu}, P^{\nu}\right]=0
$$

One can "solve" the algebra by introducing the non-canonical transformation [5, 11],

$$
r^{i}=q^{i}+\frac{j}{m^{2} c^{2}} \epsilon^{i j} p_{j} ; \quad P^{i}=p^{i}
$$


where $(q, p)$ constitute a canonical pair with the non-zero commutator $\left[q^{i}, p^{j}\right]=-i \hbar g^{i j}$. The transformation simulates the spin property of the particle, as it has originated from the non-trivial $\left[r^{i}, r^{j}\right]$ commutator in (18), which was crucial in producing the spin part of the total angular momentum. We will come to this point again. Note that although we have a canonical position coordinate $q$, the price to pay for this is that $q$ does not transform as a position vector. However, this departure can be quite small for slowly moving heavy particles.

This modifies $H$ to,

$$
\begin{gathered}
H_{\text {spin }}=H\left(P^{i}=p_{i}, \quad r^{i}=q^{i}+\frac{j}{m^{2} c^{2}} \epsilon^{i j} p_{j}\right) \\
=\frac{p^{2}}{m}-\frac{p^{4}}{4 m^{3} c^{2}}+\frac{Q^{2}}{2 \pi \epsilon_{o}}\left[\ln \frac{q}{r_{0}}(1+\alpha)+\alpha(1+\alpha)+\frac{j}{m c q} \beta\left(1-2 \alpha^{2}-\alpha \ln \frac{q}{r_{0}}\right)\right. \\
\left.+\left(\frac{j}{m c q}\right)^{2} \alpha^{2} \beta^{2}-\left(\frac{j}{m c q}\right)^{2} \alpha \beta^{2}\right],
\end{gathered}
$$

where the two dimensionless variables $\alpha$ and $\beta$ are,

$$
\alpha=\frac{\mathbf{q} \cdot \mathbf{p}}{m c q} \approx O\left(\frac{v}{c}\right) ; \quad \beta=\frac{\epsilon^{i j} q_{i} p_{j}}{m c q} \approx O\left(\frac{v}{c}\right) .
$$

Let us define,

$$
\begin{gathered}
A^{i}=\frac{Q j}{4 \pi \epsilon_{0} m c^{2} q^{2}} \epsilon^{i j} q_{j}=\sigma \frac{\epsilon^{i j} q_{j}}{q^{2}}, \\
a^{i}=\left(1-\alpha \ln \frac{q}{r_{0}}-2 \alpha^{2}\right) A^{i}
\end{gathered}
$$

and rewrite $H_{s}$ as,

$$
\begin{aligned}
H_{s}= & \frac{1}{m}(\mathbf{p}-Q \mathbf{a})^{2}+\frac{Q^{2}}{2 \pi \epsilon_{0}}\left[\ln \frac{q}{r_{0}}+\alpha\left(1+\ln \frac{q}{r_{0}}\right)+\alpha^{2}-\left(\frac{j}{m c q}\right)^{2} \alpha \beta^{2}\right. \\
& \left.+\left(\frac{j}{m c q}\right)^{2} \alpha^{2} \beta^{2}\right]-\left(\frac{Q j}{4 \pi \epsilon_{0} m c^{2}}\right)^{2} \frac{Q^{2}}{m q^{2}}\left(1-\alpha \ln \frac{q}{r_{0}}-2 \alpha^{2}\right)^{2} .
\end{aligned}
$$

The identification [11] of our system with that of a point charge interacting with Chern-Simons gauge field is now obvious. The $\alpha$-independent term in $a^{i}$ is the explicit solution of the CS gauge field. Hence we can identify 四,

$$
\theta=-\frac{Q}{\sigma}=-\frac{4 \pi \epsilon_{0} m c^{2}}{j}
$$

where $\theta$ is the CS parameter in the CS Lagrangian,

$$
L_{C S}=\frac{c \theta}{2} \int d^{2} r \epsilon^{\mu \nu \lambda} \partial_{\mu} A_{\nu} A_{\lambda} .
$$


Also the magnetic flux connected to the charged particle is $\Phi$, where

$$
\Phi=-\frac{Q j}{2 \epsilon_{0} m c^{2}} .
$$

Note the $\Phi$ is of the proper dimension of magnetic flux. This is one of our cherished results, where we have been able to obtain $\Phi$ in terms of the spinning particle parameters by simply borrowing the CS result.

Let now elaborate on the previously advertised dressing induced by the Coulomb interaction. Since we already identified our system with point charge CS system, the results of the latter can be directly used. According to CS theory [15], the physical states can be shown to be carrying an angular momentum eigenvalue $S$, which is related to the CS parameter $\theta_{C S}$ by $S=\frac{e^{2}}{4 \pi \theta_{C S}}$. Here $e$ is the fictional charge of the particle that couples to the CS gauge field to generate the anyon. This is the well known fractional spin. In our case,

$$
S=\frac{e^{2}}{4 \pi \theta_{C S}}=-\frac{Q^{2} j}{16 \pi^{2} \epsilon_{0} m c^{2}}
$$

Note that if $S=s \hbar$,

$$
s=\frac{e^{2}}{4 \pi \hbar \theta_{C S}}=\frac{e}{2 \theta_{C S}(h / e)}=\frac{\Phi}{4 \pi \Phi_{0}},
$$

where $\Phi_{0}$ is the flux quantum and $\Phi$ is obtained from (24).

However, in case of the minimal spinning particle model [5, [], due to the non-trivial $\left[r^{i}, r^{j}\right]$ commutator, the angular momentum is modified by the spin contribution in the following way,

$$
J^{\mu}=-\epsilon^{\mu \nu \lambda} r_{\nu} p_{\lambda}-\frac{j}{\sqrt{p^{2}}} p^{\mu} .
$$

Construction of the Pauli-Lubanski scalar p.J $=-j m c$ shows clearly that the particle spin is just $j$. Hence comparing with (25), we notice the extra parameters or dressings that have appeared as a result of the Coulomb interaction. This is the main result of the present work.

Let us now put the CS and our work in their proper perspectives. Our model of interacting anyons can be cast in the following form,

$$
\begin{aligned}
\mathcal{L}=\left[\Sigma _ { i = 1 } ^ { 2 } \left(\frac{1}{2} m v_{i}^{2}+\right.\right. & \left.\left.e\left(\mathbf{v}_{\mathbf{i}} \cdot \mathcal{A}-\mathcal{A}_{0}\right)\right)+\frac{c \theta_{C S}}{2} \int d^{2} r \epsilon^{\mu \nu \lambda} \mathcal{A}_{\mu} \partial_{\nu} \mathcal{A}_{\lambda}\right] \\
+ & {\left[\Sigma_{i=1}^{2} Q\left(\mathbf{v}_{\mathbf{i}} \cdot \mathbf{A}-\mathbf{A}_{\mathbf{0}}\right)\right], }
\end{aligned}
$$

where for simplicity we have used non-relativistic expressions. The fictitous charge $e$ and gauge fiels $\mathcal{A}_{\mu}$ makes the particles anyonic, and $Q$ and $A_{\mu}$ are their genuine charges and 
Coulomb interaction. For consistency, when computing $A_{\mu}$, one should consider the anyon spin as well. This will be complicated if retardation effects are to be taken into account.

On the other hand, we have started with a spinless interacting system with genuine charges and evaluated the (Darwin) Lagrangian with retardation effects duly taken care of. Subsequently we turn the whole system anyonic, (via (19)), and obtain the screening effect. The fact that our interacting anyon system, in the lowest order, is structurally similar to the CS system, has made life easier, by allowing us to borrow previous results.

We now conclude with the following comments:

(I) We have considered a system of interacting anyons, following our spinning particle model and have shown that there is a screening effect in the anyon spin, arising from mutual Coulomb interactions.

(II) We have shown how our system should be compared with the Chern- Simons construction.

(III) We have not used the $c=\hbar=1$ convention and this has made some of the relations look clumsy. We have persisted with this since all the dimensions of electromagnetic quantities have been overhauled, as we have taken the planar logarithmic Coulomb potential to be fundamental.

(IV) Unless there is a proper definition of planar $\epsilon_{0}$, with a numerical value, it is of no use to speculate about numerical estimates.

(V) Finally, it would be interesting to see if the Chern-Simons construction described above reproduces this screening. 


\section{References}

[1] J. M. Leinaas and J. Myrheim, Nuovo Cimento B37, 1(1971).

[2] F. Wilczek, Phys. Rev. Lett. 49, 957(1982).

[3] See for example F. Wilczek, Fractional Statistics and Anyon Superconductivity, (World Sc., Singapore, 1990); D. P. Arovas, R. Schrieffer, F. Wilczek and A. Zee, Nucl. Phys. B251, 117(1985); C. Hagen, Phys. Rev. D31, 2135(1985); G. W. Semenoff, Phys. Rev. Lett. 61, 517(1988).

[4] R. Jackiw and V. P. Nair, Phys. Rev. D43, 1933(1991); M. S. Plyushchay, Phys. Lett. B262, 71(1991).

[5] M. S. Plyushchay, Int. J. Mod. Phys. A7, 7045(1991); C. Chou, V. P. Nair and A. P. Polychronakos, Phys. Lett. B304, 105(1993)

[6] S. Ghosh, Phys. Lett. B338, 235(1994); Phys. Rev. D51, 5827(1995).

[7] I. V. Gorbunov, S. M. Kuzenko and S. L. Lyakhovich, preprint hep-th/9607114.

[8] S. Ghosh and S. Mukhopadhyay, Phys. Rev. D51, 6843(1995).

[9] R. Jackiw and S-Y Pi, Phys. Rev. D42, 3500(1990).

[10] Y-H Chen, F. Wilczek, E. Witten and B. I. Halperin, Int. J. Mod. Phys. B3, 1001(1989).

[11] N. Banerjee and S. Ghosh, Phys. Rev. D52, 6130(1995).

[12] J. L. Cortez and M. S. Plyushchay, preprint hep-th/9505117, (to appear in Int. J. Mod. Phys.).

[13] A. J. Hanson and T. Regge, Ann. Phys. (N.Y.) 87, 498(1974).

[14] L. D. Landau, Classical Theory of Fields, (Pergammon Press Ltd., 1975).

[15] See for example R. Jackiw, Topics in Planar Physics, 